\title{
Quantitative assessment of dietary (poly)phenol intake: a high throughput targeted metabolomics method for blood and urine samples
}

Maite Domínguez-Fernández ab, Yifan Xu a, Paul Young Tie Yang a, Wafa Alotaibi a, Rachel Gibson a, Wendy L Hall a, Leon Barronc, Iziar A. Ludwig d, Concepción Cid be and Ana Rodriguez-Mateos ${ }^{a^{*}}$

${ }^{a}$ Department of Nutritional Sciences, School of Life Course Sciences, Faculty of Life Science and Medicine, King's College London, London, UK.

b Universidad de Navarra, Facultad de Farmacia y Nutrición, Departamento de Ciencias de la Alimentación y Fisiología, C/ Irunlarrea 1, E-31008 Pamplona, Spain.

'Environmental Research Group, MRC Centre for Environment and Health, Imperial College London, London, W12 0BZ, UK.

d Program of Molecular Therapeutics, Center for Applied Medical Research (CIMA), Universidad de Navarra, Avda. Pío XII, 55, E-31008, Pamplona, Spain

e IdiSNA. Navarra Institute for Health Research, C/ Irunlarrea 1, E-31008 Pamplona, Spain.

*Address correspondence to Ana Rodriguez-Mateos, PhD, Department of Nutritional Sciences, School of Life Course Sciences, Faculty of Life Sciences and Medicine, King's College London, 150 Stamford Street, London SE1 9NH, UK. Email: ana.rodriguez-mateos@kcl.ac.uk; Tel +44 (0)20 7848 4349.

\section{SUPPORTING INFORMATION}




\section{$\underline{\text { Table of content }}$}

\section{List of standards and manufacture information}

Table S1. Summary of Metabolites Included in the Method Validation

Table S2. Concentrations (nM) at Which Precision and Accuracy were Evaluated. For Compounds with the Highest Calibration Standard or Upper Limit of Quantification (ULOQ) $\geq 400 x L O Q, 4$ Concentrations were Evaluated: LOQ (L1), Low (L2), Medium (L3) and High (L4) Level; When the ULOQ was From 200xLOQ To 43xLOQ, 3 Concentrations were Evaluated: LOQ or Low (L1 or L2), Medium (L3) and High (L4) Level; for Compounds with an ULOQ From 38xLOQ To 6xLOQ, 2 Concentrations were Evaluated: LOQ or Low (L1 or L2) and Medium (L3) Level. For Abbreviations See Table S1.

Table S3. Summary of Parameters Used for Evaluating Method Validation Specificity. RAq1 and RAq2 Difference Between the Relative Ion Abundance of q1 and q2 (Qualifier Transitions Used for Identification) in Biological Matrix (Blank Plasma and Urine Fortified with Standards) Compared with Blank Solvent. Retention Time $\left(\mathrm{T}_{\mathrm{R}}\right)$ Difference Between $\mathrm{T}_{\mathrm{R}}$ Calculated in Blank Solvent and Biological Matrix (Blank Plasma and Urine Fortified with Standards). For Abbreviations See Table S1.

Table S4. Coefficient of Variation (CV) Expressed as Percentage (\%) Between Replicates for Recovery and Matrix Effect (ME) of Compounds Studied in Plasma and Urine. For Abbreviations See Table S1.

Fig S1. LC-MS/MS chromatograms of a blank urine sample spiked with the 110 standards included in the method validation, (a) showing the original intensity in the y-axis and (b) showing the same chromatogram with the intensity zoomed $\mathrm{x} 10$.

Fig S2. LC-MS/MS chromatograms of a blank plasma sample spiked with the 119 standards included in the method validation, (a) showing the original intensity in the yaxis and (b) showing the same chromatogram with the intensity zoomed x10. 


\section{List of standards and manufacture information}

(-)-Epicatechin, hesperetin, 4-hydroxy-3-methoxybenzaldehyde, 3',4'dihydroxyphenylethanol, 4-O-caffeoylquinic acid, 4-O-feruloylquinic acid, 3-Oferuloylquinic acid, dihydroresveratrol, 3',4'-dihidroxyphenylacetic acid, 3-(2'hydroxyphenyl)propanoic acid, 3-(2',3'-dihydroxyphenyl)propanoic acid were obtained from Toronto Research Chemicals (Toronto, Canada).

(-)-Epicatechin-3'-sulfate, 3'-methoxy-(-)-epicatechin and 4'-methoxy-(-)-epicatechin were obtained from GERBU Biotechnik (Heidelberg, Germany). S-equol was obtained from Cayman Chemical (Ann Arbor, Michigan, USA). Enterolactone-glucuronide and enterolactone-sulfate ammonium was obtained from ReseaChem $\mathrm{GmbH}$ (Burgdorf, Switzerland). Equol-4'-sulfate sodium salt and equol-7-glucuronide were obtained from ChemCruz Biochemicals (Huissen, Netherlands). Kaempferol-3-glucuronide was obtained from Extrasynthese (Genay, France). 2'-, 3'- and 4'-hydroxyhippuric acids were purchased from Enamine (Kiev, Ukraine). 3,8-Dihydroxy-urolithin and 3-hydroxyurolithin were purchased from Fisher (Leicestershire, UK).

1,2-Dihydroxy-4-methylbenzene, 2-hydroxy-4/5-methylbenzene-1-sulfate, $\quad$ 2,6dihydroxybenzene-1-sulfate, 2-hydroxy-3/6-methoxybenzene-1-sulfate, 3-hydroxy-2methoxybenzene-1-sulfate, 2-hydroxybenzene-1-glucuronide and 3-methoxybenzoic acid-4-sulfate were kindly provided by Dr Cláudia Nunes dos Santos and Dr Rita Ventura, and their synthesis has been described elsewhere (Pimpao et al. 2015).

All the other polyphenol and phenolic acid aglycones and isotope labelled internal standard $\left[1,2,3-{ }^{13} \mathrm{C}\right]$ ferulic acid were obtained from Sigma-Aldrich Co (Darmstadt, Germany). All the other (poly)phenol glucuronide and sulfate conjugates were obtained from Toronto Research Chemicals (Toronto, Canada). The valerolactone was provided without information regarding the exact position of the sulfate and will be designated in this work as (4R)-5-(3'-hydroxyphenyl)- $\gamma$-valerolactone-4'-sulfate, according to the NMR spectrum provided by the supplier. 
Table S1. Summary of Metabolites Included in the Method Validation

\begin{tabular}{|c|c|c|}
\hline Recommended name & Other common names & Abbreviation \\
\hline Flavanols & Flavanols & Flavanols \\
\hline (-)-Epicatechin & - & EC \\
\hline (-)-Epicatechin-3'-sulfate & - & $\mathrm{EC}-3^{\prime}-\mathrm{S}$ \\
\hline 3'-Methoxy-(-)-epicatechin & - & 3'-ME-EC \\
\hline 4'-Methoxy-(-)-epicatechin & - & 4'-ME-EC \\
\hline Procyanidin A2 & - & Procyanidin A2 \\
\hline Flavanones & Flavanones & Flavanones \\
\hline Naringenin-4'-glucuronide & - & Naringenin-4'-GlcUA \\
\hline Naringenin-7-glucuronide & - & Naringenin-7-GlcUA \\
\hline rac-Hesperetin-3'-glucuronide & - & Hesperetin-3'-GlcUA \\
\hline rac-Hesperetin-7-sulfate & - & Hesperetin-7-S \\
\hline Hesperetin & - & Hesperetin \\
\hline Flavones & - & Flavones \\
\hline Flavone & - & Flavone \\
\hline Flavonols & Flavonols & Flavonols \\
\hline Quercetin & - & Quercetin \\
\hline Quercetin-3-sulfate & - & Quercetin-3-S \\
\hline Quercetin-3-glucuronide & - & Quercetin-3-GlcUA \\
\hline Quercetin-7-glucuronide & - & Quercetin-7-GlcUA \\
\hline Kaempferol-3-glucuronide & - & Kaempferol-3-GlcUA \\
\hline Myricetin & - & Myricetin \\
\hline Morin & - & Morin \\
\hline Dihydrochalcones & Dihydrochalcones & Dihydrochalcones \\
\hline Phloretin & - & Phloretin \\
\hline Isoflavones & Isoflavonoids & Isoflavonoids \\
\hline Equol & - & Equol \\
\hline Equol-7-glucuronide & - & Equol-7-GlcUA \\
\hline Equol-4'-sulfate & - & Equol-4'-S \\
\hline Daidzein & - & Daidzein \\
\hline Lignans & Lignans & Lignans \\
\hline Secoisolariciresinol & - & SECO \\
\hline Enterodiol & - & Enterodiol \\
\hline Enterolactone-glucuronide & - & Enterolactone-GlcUA \\
\hline Enterolactone-sulfate & - & Enterolactone-S \\
\hline Enterolactone & - & Enterolactone \\
\hline Benzene diols and triols & Benzene diols and triols & Benzene diols and triols \\
\hline 1,2-Dihydroxy-4-methylbenzene & 4-Methylcatechol & 1,2-diOH-4-Me-Benz \\
\hline 2-Hydroxy-4-methylbenzene-1-sulfate & 4-Methylcatechol-1-sulfate & 2-OH-4-Me-Benz-1-S \\
\hline 2-Hydroxy-5-methylbenzene-1-sulfate & 4-Methylcatechol-2-sulfate & 2-OH-5-Me-Benz-1-S \\
\hline 2-Hydroxybenzene-1-glucuronide & Catechol-1-glucuronide & 2-OH-Benz-1-GlcUA \\
\hline 2,6-Dihydroxybenzene-1-sulfate & Pyrogallol-2-sulfate & 2,6-diOH-Benz-1-S \\
\hline 2-Hydroxy-3-methoxybenzene-1-sulfate & 1-Methylpyrogallol-3-sulfate & 2-OH-3-meO-Benz-1-S \\
\hline 2-Hydroxy-6-methoxybenzene-1-sulfate & 1-Methylpyrogallol-2-sulfate & 2-OH-6-meO-Benz-1-S \\
\hline 3-Hydroxy-2-methoxybenzene-1-sulfate & 2-Methylpyrogallol-1-sulfate & 3-OH-2-meO-Benz-1-S \\
\hline Benzaldehydes & Benzaldehydes & Benzaldehydes \\
\hline 3,4-Dihydroxybenzaldehyde & - & 3,4-diOH-BAL \\
\hline 4-Hydroxybenzaldehyde & - & 4-OH-BAL \\
\hline 4-Hydroxy-3-methoxybenzaldehyde & Vanillin & 4-OH-3-meO-BAL \\
\hline Hydroxycoumarins & Hydroxycoumarins & Hydroxycoumarins \\
\hline 7,8-Dihydroxycoumarin & & 7,8-diOH-CM \\
\hline 3,8-Dihydroxy-urolithin & Urolithin A & 3,8-diOH-Uro \\
\hline 3-Hydroxy-urolithin & Urolithin B & 3-OH-Uro \\
\hline Tyrosols & Tyrosols & Tyrosols \\
\hline Phenylethanol-4'-sulfate & Tyrosol sulfate & ph-etOH-4'-S \\
\hline 3',4'-Dihydroxyphenylethanol & Hydroxytyrosol & $3^{\prime}, 4^{\prime}$-diOH-ph-etOH \\
\hline 3'-Hydroxyphenylethanol-4'-sulfate & Hydroxytyrosol-4'-sulfate & 3'-OH-ph-etOH-4'-S \\
\hline 4'-Hydroxyphenylethanol-3'-sulfate & Hydroxytyrosol-3'-sulfate & 4'-OH-ph-etOH-3'-S \\
\hline Benzoic acids & Benzoic acids & Benzoic acids \\
\hline Benzoic acid & - & $\mathrm{BA}$ \\
\hline 2-Hydroxybenzoic acid & - & 2-OH-BA \\
\hline 3-Hydroxybenzoic acid & - & $3-\mathrm{OH}-\mathrm{BA}$ \\
\hline 4-Hydroxybenzoic acid & - & 4-OH-BA \\
\hline 2,3-Dihydroxybenzoic acid & - & 2,3-diOH-BA \\
\hline 2,4-Dihydroxybenzoic acid & - & 2,4-diOH-BA \\
\hline
\end{tabular}




\begin{tabular}{|c|c|c|}
\hline 2,5-Dihydroxybenzoic acid & - & 2,5-diOH-BA \\
\hline 2,6-Dihydroxybenzoic acid & - & 2,6-diOH-BA \\
\hline 3,4-Dihydroxybenzoic acid & Protocatechuic acid & 3,4-diOH-BA \\
\hline 3,5-Dihydroxybenzoic acid & - & 3,5-diOH-BA \\
\hline 2,3,4-Trihydroxybenzoic acid & - & 2,3,4-triOH-BA \\
\hline 2-Hydroxy-4-methoxybenzoic acid & - & 2-OH-4-methO-BA \\
\hline 3-Hydroxybenzoic acid-4-sulfate & Protocatechuic acid-4-sulfate & 3-OH-BA-4-S \\
\hline 4-Hydroxybenzoic acid-3-sulfate & Protocatechuic acid-3-sulfate & 4-OH-BA-3-S \\
\hline 4-Hydroxybenzoic acid-3-glucuronide & Protocatechuic acid-3-glucuronide & 4-OH-BA-3-GlcUA \\
\hline 4-Hydroxy-3,5-dimethoxybenzoic acid & Syringic acid & 4-OH-3,5-dimeO-BA \\
\hline 3,4,5-Trihydroxybenzene ethyl ester & Ethyl gallate & 3,4,5-triOH-Benz-OEt \\
\hline 3,4,5-Trihydroxybenzoic acid & Gallic acid & 3,4,5-triOH-BA \\
\hline 3-Hydroxy-4-methoxybenzoic acid-5-sulfate & 4-Methylgallic acid-3-sulfate & 3-OH-4-meO-BA-5-S \\
\hline 4-Hydroxy-3-methoxybenzoic acid & Vanillic acid & 4-OH-3-meO-BA \\
\hline 3-Methoxybenzoic acid-4-sulfate & Vanillic acid-4-sulfate & 3-meO-BA-4-S \\
\hline 4-Methoxybenzoic acid-3-sulfate & Isovanillic acid-3-sulfate & 4-meO-BA-3-S \\
\hline Hippuric acids & Hippuric acids & Hippuric acids \\
\hline Hippuric acid & - & HA \\
\hline 2'-Hydroxyhippuric acid & Salicyluric acid & $2^{\prime}-\mathrm{OH}-\mathrm{HA}$ \\
\hline 3'-Hydroxyhippuric acid & - & 3'-OH-HA \\
\hline 4'-Hydroxyhippuric acid & - & $4^{\prime}-\mathrm{OH}-\mathrm{HA}$ \\
\hline alpha-hydroxyhippuric acid & - & $\alpha-\mathrm{OH}-\mathrm{HA}$ \\
\hline Cinnamic acids & Cinnamic acids & Cinnamic acids \\
\hline Cinnamic acid & - & $\mathrm{CA}$ \\
\hline 3',4'-Dihydroxycinnamic acid & Caffeic acid & 3',4'-diOH-CA \\
\hline 3'-Hydroxycinnamic acid-4'-sulfate & Caffeic acid-4'-sulfate & 3'-OH-CA-4'-S \\
\hline 4'-Hydroxycinnamic acid-3'-sulfate & Caffeic acid-3'-sulfate & 4'-OH-CA-3'-S \\
\hline 3'-Hydroxycinnamic acid-4'-glucuronide & Caffeic acid-4'-glucuronide & 3'-OH-CA-4'-GlcUA \\
\hline 4'-Hydroxycinnamic acid-3'-glucuronide & Caffeic acid-3'-glucuronide & 4'-OH-CA-3'-GlcUA \\
\hline 4'-Hydroxy-3'-methoxycinnamic acid & trans-Ferulic acid & 4'-OH-3'-meO-CA \\
\hline 3'-Methoxycinnamic acid-4'-sulfate & Ferulic acid-4'-sulfate & 3'-meO-CA-4'-S \\
\hline 3'-Methoxycinnamic acid-4'-glucuronide & Ferulic acid-4'-glucuronide & 3'-meO-CA-4'-GlcUA \\
\hline 3'-Hydroxy-4'-methoxycinnamic acid & Isoferulic acid & 3'-OH-4'-methO-CA \\
\hline 4'-Methoxycinnamic acid-3'-sulfate & Isoferulic acid-3'-sulfate & 4'-meO-CA-3'-S \\
\hline 4'-Methoxycinnamic acid-3'-glucuronide & Isoferulic acid-3'-glucuronide & 4'-meO-CA-3'-GlcUA \\
\hline 4-O-Caffeoylquinic acid & Cryptochlorogenic acid & 4-CQA \\
\hline 5-O-Caffeoylquinic acid & Chlorogenic acid & 5-CQA \\
\hline 3-O-Feruloylquinic acid & - & 3-FQA \\
\hline 4-O-Feruloylquinic acid & - & 4-FQA \\
\hline 4'-Hydroxy-3',5'-dimethoxycinnamic acid & Sinapic acid & 4'-OH-3',5'-dimeO-CA \\
\hline 3'-Hydroxycinnamic acid & $m$-Coumaric acid & 3'-OH-CA \\
\hline 4'-Hydroxycinnamic acid & $p$-Coumaric acid & $4^{\prime}-\mathrm{OH}-\mathrm{CA}$ \\
\hline 2'-Hydroxycinnamic acid & $o$-Coumaric acid & $2^{\prime}-\mathrm{OH}-\mathrm{CA}$ \\
\hline Cinnamic acid-4'-sulfate & p-Coumaric acid-4'-sulfate & $\mathrm{CA}-4^{\prime}-\mathrm{S}$ \\
\hline Cinnamic acid-4'-glucuronide & p-Coumaric acid-4'-glucuronide & CA-4'-GlcUA \\
\hline Phenylacetic acids & Phenylacetic acids & Phenylacetic acids \\
\hline Phenylacetic acid & - & $\mathrm{PA}$ \\
\hline 3'-Hydroxyphenylacetic acid & - & 3'-OH-PA \\
\hline 3',4'-Dihidroxyphenylacetic acid & Homoprotocatechuic acid & $3^{\prime}, 4^{\prime}-\mathrm{diOH}-\mathrm{PA}$ \\
\hline 3'-Methyoxyphenylacetic acid-4'-sulfate & Homovanillic acid-sulfate & 3'-meO-PA-4'-S \\
\hline Phenylpropanoic acids & Phenylpropanoic acids & Phenylpropanoic acids \\
\hline 2-(4'-Hydroxyphenoxy)propanoic acid & - & 2-(4'-OH-ph-O)-PrA \\
\hline 3-(2'-Hydroxyphenyl)propanoic acid & - & $3-\left(2^{\prime}-\mathrm{OH}-\mathrm{ph}\right)-\mathrm{PrA}$ \\
\hline 3-(3'-Hydroxyphenyl)propanoic acid & - & 3-(3'-OH-ph)-PrA \\
\hline 3-(2',3'-Dihydroxyphenyl)propanoic acid & - & $3-\left(2^{\prime}, 3^{\prime}-\mathrm{diOH}-\mathrm{ph}\right)-\mathrm{PrA}$ \\
\hline 3-(2',4'-Dihydroxyphenyl)propanoic acid & - & $3-\left(2^{\prime}, 4^{\prime}-\mathrm{diOH}-\mathrm{ph}\right)-\mathrm{PrA}$ \\
\hline 3-(3',4'-Dihydroxyphenyl)propanoic acid & Dihydrocaffeic acid & 3-(3',4'-diOH-ph)-PrA \\
\hline 3-(3',5'-Dihydroxyphenyl)propanoic acid & - & $3-\left(3^{\prime}, 5^{\prime}-\mathrm{diOH}-\mathrm{ph}\right)-\mathrm{PrA}$ \\
\hline 2-Hydroxy-3-(4'-hydroxyphenyl)propanoic acid & - & $3-\left(4^{\prime}-\mathrm{OH}-\mathrm{ph}\right)-2-\mathrm{OH}-\mathrm{PrA}$ \\
\hline 3-(4'-Hydroxy-3'-methoxyphenyl)propanoic acid & Dihydroferulic acid & 3-(4'-OH-3'-meO-ph)-PrA \\
\hline 3-(4'-Hydroxyphenyl)propanoic acid-3'-glucuronide & Dihydrocaffeic acid-3'-glucuronide & 3-(4'-OH-ph)-PrA-3'-GlcUA \\
\hline 3-(4'-Hydroxyphenyl)propanoic acid-3'-sulfate & Dihydrocaffeic acid-3'-sulfate & 3-(4'-OH-ph)-PrA-3'-S \\
\hline 3-(3'-Methoxyphenyl)propanoic acid-4'-sulfate & Dihydroferulic acid-4'-sulfate & 3-(3'-meO-ph)-PrA-4'-S \\
\hline 3-(3'-Methoxyphenyl)propanoic acid-4'-glucuronide & Dihydroferulic acid-4'-glucuronide & 3-(3'-meO-ph)-PrA-4'-GlcUA \\
\hline 3-(4'-Methoxyphenyl)propanoic acid-3'-sulfate & Dihydroisoferulic acid-3'-sulfate & $3-\left(4^{\prime}-\mathrm{meO}-\mathrm{ph}\right)-\mathrm{PrA}-3^{\prime}-\mathrm{S}$ \\
\hline $\begin{array}{l}\text { 3-(4'-Methoxyphenyl)propanoic acid-3'-glucuronide } \\
\text { Valerolactones }\end{array}$ & $\begin{array}{l}\text { Dihydroisoferulic acid-3'-glucuronide } \\
\text { Valerolactones }\end{array}$ & $\begin{array}{c}\text { 3-(4'-meO-ph)-PrA-3'-GlcUA } \\
\text { Valerolactones }\end{array}$ \\
\hline
\end{tabular}




\begin{tabular}{ccc}
\hline (4R)-5-(3'-hydroxyphenyl)- $\gamma$-valerolactone-4'-sulfate & - & $5-\left(3^{\prime}-\mathrm{OH}-\mathrm{ph}\right)-\gamma-\mathrm{VL}-4^{\prime}-\mathrm{S}$ \\
Stilbenes & Stillbenes \\
Dihydroresveratrol & - & diOH-res \\
trans-Resveratrol-4'-glucuronide & - & t-Res-4'-GlcUA \\
trans-Resveratrol-3-glucuronide & - & t-Res-3-GlcUA \\
cis-Resveratrol-4'-glucuronide & - & c-Res-4'-GlcUA \\
cis-Resveratrol-3-glucuronide & - & t-Res-3-S \\
trans-Resveratrol-3-Sulfate & - & Internal standards \\
Internal standards & Internal standards & Taxifolin \\
Taxifolin & - & {$\left[1,2,3-{ }^{3} \mathrm{C}\right] \mathrm{FA}$} \\
\hline $\left.1,2,3-{ }^{13} \mathrm{C}\right]$ ferulic acid & - &
\end{tabular}


Table S2. Concentrations (nM) at Which Precision and Accuracy were Evaluated. For Compounds with the Highest Calibration Standard or Upper Limit of Quantification (ULOQ) $\geq 400 x L O Q, 4$ Concentrations were Evaluated: LOQ (L1), Low (L2), Medium (L3) and High (L4) Level; When the ULOQ was From 200xLOQ To 43xLOQ, 3 Concentrations were Evaluated: LOQ or Low (L1 or L2), Medium (L3) and High (L4) Level; for Compounds with an ULOQ From 38xLOQ To 6xLOQ, 2 Concentrations were Evaluated: LOQ or Low (L1 or L2) and Medium (L3) Level. For Abbreviations See Table S1.

\begin{tabular}{|c|c|c|c|c|}
\hline Compounds & L1 & L2 & L3 & L4 \\
\hline \multicolumn{5}{|l|}{$\begin{array}{l}\text { Flavanols } \\
\text {. }\end{array}$} \\
\hline $\mathrm{EC}$ & 3.9 & 26.9 & 511.5 & 1153.9 \\
\hline EC-3'-S & 26.2 & - & 498.6 & 1124.7 \\
\hline 3'-ME-EC & 26.5 & - & 504.3 & 1137.6 \\
\hline 4'-ME-EC & 3.8 & 26.5 & 504.3 & 1137.6 \\
\hline Procyanidin A2 & 7.7 & 54.2 & 1029.3 & 2321.6 \\
\hline \multicolumn{5}{|l|}{ Flavanones } \\
\hline Naringenin-4'-GlcUA & 3.8 & 26.4 & 502.0 & 1132.3 \\
\hline Naringenin-7-GlcUA & 3.8 & 26.4 & 502.0 & 1132.3 \\
\hline Hesperetin-3'-GlcUA & 3.9 & 27.0 & 513.3 & 1157.7 \\
\hline Hesperetin-7-S & 26.6 & - & 506.1 & 1141.5 \\
\hline Hesperetin & 3.9 & 27.0 & 512.6 & 1156.2 \\
\hline \multicolumn{5}{|l|}{ Flavones } \\
\hline Flavone & - & 26.7 & 506.4 & 1142.2 \\
\hline \multicolumn{5}{|l|}{ Flavonols } \\
\hline Quercetin & 0.8 & 5.3 & 101.2 & 228.3 \\
\hline Quercetin-3-S & 7.7 & 53.9 & 1023.7 & 2309.0 \\
\hline Quercetin-3-GlcUA & 0.8 & 5.6 & 106.9 & 241.2 \\
\hline Quercetin-7-GlcUA & 3.9 & 27.0 & 513.3 & 1157.8 \\
\hline Kaempferol-3-GlcUA & 0.8 & 5.8 & 110.6 & 249.6 \\
\hline Myricetin & 3.8 & 26.8 & 509.9 & 1150.2 \\
\hline Morin & 27.6 & - & 524.7 & 1183.5 \\
\hline \multicolumn{5}{|l|}{ Dihydrochalcones } \\
\hline Phloretin & 7.7 & 53.7 & 1020.6 & 2302.1 \\
\hline \multicolumn{5}{|l|}{ Isoflavones } \\
\hline Equol & 26.4 & - & 501.7 & 1131.6 \\
\hline Equol-7-GlcUA & 3.8 & 26.9 & 511.1 & 1152.8 \\
\hline Equol-4'-S & 3.8 & 26.9 & 511.1 & 1152.8 \\
\hline Daidzein & 3.6 & 25.4 & 483.4 & 1090.3 \\
\hline \multicolumn{5}{|l|}{ Lignans } \\
\hline SECO & 26.7 & - & 508.2 & 1146.2 \\
\hline Enterodiol & 3.8 & 26.7 & 507.5 & 1144.7 \\
\hline Enterolactone-GlcUA & - & 506.7 & 1142.9 & - \\
\hline Enterolactone-S & 3.9 & 27.0 & 512.4 & - \\
\hline Enterolactone & 26.6 & - & 506.2 & 1141.9 \\
\hline \multicolumn{5}{|l|}{ Benzene diols and triols } \\
\hline 1,2-diOH-4-Me-Benz & 27.2 & - & 516.7 & 1165.6 \\
\hline 2-OH-4/5-Me-Benz-1-S* & - & 269.8 & 5125.4 & 11561.1 \\
\hline 2-OH-Benz-1-GlcUA & 3.9 & 27.3 & 518.3 & 1169.0 \\
\hline 2,6-diOH-Benz-1-S & 5.1 & - & 97.6 & 220.1 \\
\hline 2-OH-3/6-meO-Benz-1-S* & - & 54.0 & 1026.8 & 2316.1 \\
\hline 3-OH-2-meO-Benz-1-S & 26.4 & - & 501.8 & - \\
\hline \multicolumn{5}{|l|}{ Benzaldehydes } \\
\hline 3,4-diOH-BAL & 25.0 & - & 475.5 & 1072.7 \\
\hline 4-OH-BAL & 25.8 & - & 489.3 & 1103.6 \\
\hline 4-OH-3-meO-BAL & 7.3 & 50.8 & 964.9 & 2176.5 \\
\hline \multicolumn{5}{|l|}{ Hydroxycoumarins } \\
\hline 7,8-diOH-CM & - & 516.9 & 1165.9 & - \\
\hline 3,8-diOH-Uro & 3.9 & 27.1 & 515.6 & 1162.9 \\
\hline 3-OH-Uro & 4.0 & 27.9 & 530.3 & 1196.2 \\
\hline \multicolumn{5}{|l|}{ Tyrosols } \\
\hline ph-etOH-4'-S & - & 26.0 & 494.1 & 1114.4 \\
\hline $3^{\prime}, 4^{\prime}$-diOH-ph-etOH & - & 26.4 & 501.7 & 1131.7 \\
\hline 3'-OH-ph-etOH-4'-S & 3.8 & 26.9 & 511.1 & - \\
\hline 4'-OH-ph-etOH-3'-S & 3.9 & 27.3 & 519.1 & 1170.9 \\
\hline \multicolumn{5}{|l|}{ Benzoic acids } \\
\hline BA & 38.7 & 270.9 & 5146.8 & 11609.2 \\
\hline 2-OH-BA & 27.0 & - & 512.1 & 1155.0 \\
\hline 3-OH-BA & 53.9 & - & 1024.5 & 2311.0 \\
\hline 4-OH-BA & - & 27.0 & 512.1 & 1155.0 \\
\hline 2,3-diOH-BA & 38.5 & 269.3 & 5115.7 & 11539.1 \\
\hline 2,4/2,6-diOH-BA* & 12.1 & 84.7 & 1609.1 & 3629.5 \\
\hline 2,5-diOH-BA & 7.7 & 54.0 & 1025.9 & 2314.1 \\
\hline 2,6-diOH-BA & - & 29.9 & 568.2 & 1281.7 \\
\hline 3,4-diOH-BA & 26.9 & - & 511.6 & 1153.9 \\
\hline 3,5-diOH-BA & - & 55.0 & 1045.5 & 2358.3 \\
\hline 2,3,4-triOH-BA & 26.3 & - & 500.4 & 1128.6 \\
\hline 2-OH-4-meO-BA & 3.8 & 26.9 & 510.7 & 1151.8 \\
\hline
\end{tabular}




\begin{tabular}{|c|c|c|c|c|}
\hline 3-OH-BA-4-S & 3.8 & 26.8 & 509.7 & 1149.7 \\
\hline 4-OH-BA-3-S & 3.8 & 26.8 & 509.7 & 1149.7 \\
\hline 4-OH-BA-3-GlcUA & - & 27.0 & 512.1 & 1155.1 \\
\hline 4-OH-3,5-dimeO-BA & - & 102.8 & 231.8 & - \\
\hline 3,4,5-triOH-Benz-OEt & 1.0 & 6.7 & 126.5 & 285.3 \\
\hline $3,4,5$-triOH-BA & 7.2 & - & 136.0 & 306.8 \\
\hline 3-OH-4-meO-BA-5-S & 3.8 & 26.5 & 503.4 & 1135.5 \\
\hline 4-OH-3-meO-BA & - & 511.0 & 1152.6 & - \\
\hline 3-meO-BA-4-S & 3.8 & 26.8 & 509.0 & 1148.2 \\
\hline 4-meO-BA-3-S & 3.9 & 27.2 & 517.3 & 1166.9 \\
\hline Hippuric acids & - & - & - & - \\
\hline $\mathrm{HA}$ & - & 536.9 & 10200.1 & 23007.7 \\
\hline $2^{\prime}-\mathrm{OH}-\mathrm{HA}$ & 5.5 & - & 104.8 & 236.5 \\
\hline 3'-OH-HA & 4.0 & 28.2 & 535.0 & 1206.8 \\
\hline 4'-OH-HA & - & 25.6 & 486.8 & 1098.0 \\
\hline$\alpha-\mathrm{OH}-\mathrm{HA}$ & 19.3 & 135.4 & 2572.8 & 5803.4 \\
\hline \multicolumn{5}{|l|}{ Cinnamic acids } \\
\hline $\mathrm{CA}$ & 53.2 & - & 1010.2 & 2278.7 \\
\hline 3',4'-diOH-CA & 5.7 & - & 107.6 & 242.7 \\
\hline $3^{\prime}-\mathrm{OH}-\mathrm{CA}-4^{\prime}-\mathrm{S}$ & 3.8 & 26.9 & 511.1 & 1152.9 \\
\hline $4^{\prime}-\mathrm{OH}-\mathrm{CA}-3^{\prime}-\mathrm{S}$ & 3.8 & 26.9 & 511.1 & 1152.9 \\
\hline 3'-OH-CA-4'-GlcUA & 0.8 & 5.3 & 100.5 & 226.7 \\
\hline 4'-OH-CA-3'-GlcUA & 0.8 & 5.3 & 100.5 & 226.7 \\
\hline $4^{\prime}-\mathrm{OH}-3^{\prime}-\mathrm{meO}-\mathrm{CA}$ & - & 1023.9 & 2309.6 & - \\
\hline 3'-meO-CA-4'-S & 7.7 & 53.8 & 1022.4 & 2306.2 \\
\hline 3'-meO-CA-4'-GlcUA & - & 27.8 & 528.3 & 1191.5 \\
\hline 3'-OH-4'-meO-CA & 38.5 & 269.2 & 5115.3 & 11538.2 \\
\hline $4^{\prime}-\mathrm{meO}-\mathrm{CA}-3^{\prime}-\mathrm{S}$ & 3.7 & 26.1 & 495.1 & 1116.8 \\
\hline 4'-meO-CA-3'-GlcUA & 27.6 & - & 524.9 & 1184.0 \\
\hline 4-CQA & 4.7 & - & 88.4 & 199.3 \\
\hline 4/5-CQA* & 10.0 & - & 190.6 & 430.0 \\
\hline 3-FQA & 0.8 & 5.6 & 105.6 & 238.1 \\
\hline 4-FQA & 0.8 & 5.3 & 101.1 & 228.1 \\
\hline $4^{\prime}-\mathrm{OH}-3^{\prime}, 5^{\prime}$-dimeO-CA & 0.8 & 5.4 & 101.9 & 229.9 \\
\hline 3'-OH-CA & - & 88.9 & 200.6 & - \\
\hline 4'-OH-CA & 5.4 & - & 102.3 & 230.7 \\
\hline 2'-OH-CA & - & 3.9 & 74.2 & 167.4 \\
\hline CA-4'-S & 3.9 & 27.5 & 521.9 & 1177.2 \\
\hline CA-4'-GlcUA & 3.9 & 27.0 & 512.6 & 1156.3 \\
\hline \multicolumn{5}{|l|}{ Phenylacetic acids } \\
\hline $\mathrm{PA}$ & 76.7 & 536.7 & 10196.8 & 23000.3 \\
\hline 3'-OH-PA & - & 1024.6 & 2311.2 & - \\
\hline 3',4'-diOH-PA & 7.7 & 53.7 & 1019.7 & 2300.0 \\
\hline 3'-meO-PA-4'-S & 0.8 & 5.3 & 100.2 & 226.1 \\
\hline \multicolumn{5}{|l|}{ Phenylpropionic acids } \\
\hline 2-(4'-OH-ph-O)-PrA & - & 53.7 & 1020.7 & 2302.2 \\
\hline 3-(2'-OH-ph)-PrA & 3.7 & 25.9 & 492.5 & 1111.0 \\
\hline $3-\left(3^{\prime}-\mathrm{OH}-\mathrm{ph}\right)-\mathrm{PrA}$ & 3.9 & 27.4 & 520.8 & 1174.8 \\
\hline $3-\left(2^{\prime}, 3^{\prime}-\mathrm{diOH}-\mathrm{ph}\right)-\mathrm{PrA}$ & 3.5 & 24.1 & 458.3 & 1033.7 \\
\hline $3-\left(2^{\prime}, 4^{\prime}-\mathrm{diOH}-\mathrm{ph}\right)-\mathrm{PrA}$ & 30.3 & - & 575.7 & 1298.5 \\
\hline $3-\left(3^{\prime}, 4^{\prime}-\mathrm{diOH}-\mathrm{ph}\right)-\mathrm{PrA}$ & 26.9 & - & 510.3 & 1151.1 \\
\hline $3-\left(3^{\prime}, 5^{\prime}-\mathrm{diOH}-\mathrm{ph}\right)-\mathrm{PrA}$ & 4.5 & 31.3 & 595.3 & 1342.8 \\
\hline 3-(4'-OH-ph)-2-OH-PrA & - & 578.5 & 1304.8 & - \\
\hline 3-(4'-OH-3'-meO-ph)-PrA & - & 513.7 & 1158.7 & - \\
\hline 3-(4'-OH-ph)-PrA-3'-GlcUA & 8.5 & 59.6 & 1132.2 & 2553.9 \\
\hline $3-\left(4^{\prime}-\mathrm{OH}-\mathrm{ph}\right)-\mathrm{PrA}-3^{\prime}-\mathrm{S}$ & 3.9 & 27.3 & 517.9 & 1168.2 \\
\hline 3-(3'-meO-ph)-PrA-4'-S & - & 5.7 & 107.4 & 242.1 \\
\hline 3-(3'-meO-ph)-PrA-4'-GlcUA & 5.1 & - & 96.2 & 216.9 \\
\hline $3-\left(4^{\prime}-\mathrm{meO}-\mathrm{ph}\right)-\mathrm{PrA}-3^{\prime}-\mathrm{S}$ & 0.8 & 5.3 & 99.7 & 224.8 \\
\hline 3-(4'-meO-ph)-PrA-3'-GlcUA & 5.1 & - & 96.2 & 216.9 \\
\hline Valerolactones & - & - & - & - \\
\hline $5-\left(3^{\prime}-\mathrm{OH}-\mathrm{ph}\right)-\gamma-\mathrm{VL}-4^{\prime}-\mathrm{S}$ & - & 511.1 & 1152.9 & - \\
\hline \multicolumn{5}{|l|}{ Stilbenes } \\
\hline diOH-res & 3.9 & 27.1 & 515.4 & 1162.6 \\
\hline t-Res-4'-GlcUA & 3.8 & 26.8 & 510.0 & 1150.4 \\
\hline t-Res-3-GlcUA & 3.8 & 26.8 & 510.0 & 1150.4 \\
\hline c-Res-4'/3-GlcUA* & 7.7 & 53.9 & 1024.1 & 2310.0 \\
\hline c-Res-3-GlcUA & 3.9 & 27.0 & 513.1 & 1157.3 \\
\hline t-Res-3-S & 3.9 & 27.1 & 514.2 & 1159.8 \\
\hline
\end{tabular}

*Mix of two isomers 
Table S3. Summary of Parameters Used for Evaluating Method Validation Specificity. RAq1 and RAq2 Difference Between the Relative Ion Abundance of q1 and q2 (Qualifier Transitions Used for Identification) in Biological Matrix (Blank Plasma and Urine Fortified with Standards) Compared with Blank Solvent. Retention Time $\left(\mathrm{T}_{\mathrm{R}}\right)$ Difference Between $T_{R}$ Calculated in Blank Solvent and Biological Matrix (Blank Plasma and Urine Fortified with Standards). For Abbreviations See Table S1.

\begin{tabular}{|c|c|c|c|c|c|c|}
\hline \multirow[b]{2}{*}{ Compounds } & \multicolumn{3}{|c|}{ Urine } & \multicolumn{3}{|c|}{ Plasma } \\
\hline & $\begin{array}{c}\text { RAq1 } \\
\text { difference }(\%)\end{array}$ & $\begin{array}{c}\text { RAq2 } \\
\text { difference (\%) }\end{array}$ & $\begin{array}{c}\mathrm{T}_{\mathrm{R}} \text { difference } \\
(\mathrm{min})\end{array}$ & $\begin{array}{c}\text { RAq1 } \\
\text { difference }(\%)\end{array}$ & $\begin{array}{c}\text { RAq2 } \\
\text { difference }(\%)\end{array}$ & $\begin{array}{c}\mathrm{T}_{\mathrm{R}} \text { difference } \\
(\mathrm{min})\end{array}$ \\
\hline \multicolumn{7}{|l|}{ Flavanols } \\
\hline $\mathrm{EC}$ & 13.25 & 0.08 & 0.00 & 2.12 & 4.34 & 0.00 \\
\hline EC-3'-S & 3.25 & 0.88 & 0.02 & 0.22 & 8.08 & 0.00 \\
\hline 3'-ME-EC & 19.51 & 5.13 & 0.05 & 19.49 & 12.37 & 0.00 \\
\hline 4'-ME-EC & 4.49 & - & 0.00 & 2.65 & - & 0.00 \\
\hline Procyanidin A2 & 2.92 & - & 0.00 & 1.20 & - & 0.00 \\
\hline \multicolumn{7}{|l|}{ Flavanones } \\
\hline Naringenin-4'-GlcUA & - & - & 0.12 & - & - & 0.13 \\
\hline Naringenin-7-GlcUA & - & - & 0.00 & - & - & 0.00 \\
\hline Hesperetin-3'-GlcUA & 4.50 & 1.74 & -0.03 & 3.07 & 4.36 & 0.00 \\
\hline Hesperetin-7-S & 18.79 & 18.68 & 0.00 & 12.72 & 13.03 & 0.00 \\
\hline Hesperetin & 4.87 & 7.36 & 0.00 & 6.52 & 0.33 & 0.00 \\
\hline \multicolumn{7}{|l|}{ Flavones } \\
\hline Flavone & 3.62 & 4.39 & 0.00 & 9.64 & 4.51 & 0.00 \\
\hline \multicolumn{7}{|l|}{ Flavonols } \\
\hline Quercetin & 17.97 & 16.70 & 0.00 & 16.13 & 19.60 & 0.00 \\
\hline Quercetin-3-S & 3.67 & - & -0.08 & 0.81 & - & 0.00 \\
\hline Quercetin-3-GlcUA & - & - & 0.04 & - & - & 0.00 \\
\hline Quercetin-7-GlcUA & - & - & 0.03 & - & - & 0.00 \\
\hline Kaempferol-3-GlcUA & - & - & 0.00 & - & - & 0.00 \\
\hline Myricetin & 1.54 & 3.90 & 0.06 & 6.83 & 6.32 & 0.00 \\
\hline Morin & 11.81 & 6.83 & 0.10 & 1.79 & 3.98 & 0.09 \\
\hline \multicolumn{7}{|l|}{ Dihydrochalcones } \\
\hline Phloretin & 2.59 & 1.82 & 0.00 & 4.28 & 3.01 & 0.00 \\
\hline \multicolumn{7}{|l|}{ Isoflavones } \\
\hline Equol & 9.09 & 8.37 & 0.00 & 10.88 & 16.87 & 0.00 \\
\hline Equol-7-GlcUA & 0.19 & 1.52 & 0.00 & 0.52 & 2.75 & 0.00 \\
\hline Equol-4'-S & 1.77 & 1.07 & 0.04 & 6.30 & 6.99 & -0.08 \\
\hline Daidzein & 1.00 & 3.15 & -0.03 & 1.47 & 1.97 & 0.00 \\
\hline \multicolumn{7}{|l|}{ Lignans } \\
\hline SECO & 5.75 & 1.61 & 0.00 & 17.44 & 17.31 & 0.00 \\
\hline Enterodiol & 1.92 & 5.21 & 0.03 & 6.44 & 5.16 & 0.00 \\
\hline Enterolactone-GlcUA & 13.43 & 1.05 & 0.03 & 4.15 & 6.23 & 0.00 \\
\hline Enterolactone-S & 11.82 & 13.26 & 0.03 & 15.55 & 18.06 & 0.00 \\
\hline Enterolactone & 0.92 & 2.61 & 0.00 & 1.49 & 2.25 & 0.00 \\
\hline \multicolumn{7}{|l|}{ Benzene diols and triols } \\
\hline 1,2-diOH-4-Me-Benz & 13.35 & - & 0.00 & 13.80 & - & 0.00 \\
\hline 2-OH-4/5-Me-Benz-1-S* & 1.70 & 2.33 & 0.00 & 0.52 & 0.85 & 0.00 \\
\hline 2-OH-Benz-1-GlcUA & 4.18 & - & 0.04 & 0.55 & - & 0.00 \\
\hline 2,6-diOH-Benz-1-S & 2.59 & 1.92 & 0.20 & 8.46 & 12.42 & 0.00 \\
\hline 2-OH-3/6-meO-Benz-1-S* & - & - & -0.07 & 8.79 & - & -0.04 \\
\hline 3-OH-2-meO-Benz-1-S & 19.88 & - & 0.20 & - & - & 0.00 \\
\hline Benzaldehydes & & & & & & \\
\hline 3,4-diOH-BAL & 14.12 & 9.92 & 0.00 & 0.14 & 2.79 & 0.00 \\
\hline 4-OH-BAL & 7.74 & - & 0.00 & 5.07 & - & 0.00 \\
\hline 4-OH-3-meO-BAL & 6.81 & 10.94 & 0.00 & 3.15 & 2.32 & 0.00 \\
\hline Hydroxycoumarins & & & & & & \\
\hline 7,8-diOH-CM & 1.33 & 5.38 & 0.00 & 3.57 & 11.68 & 0.00 \\
\hline 3,8-diOH-Uro & 1.11 & - & 0.00 & 3.90 & - & 0.00 \\
\hline 3-OH-Uro & 7.22 & 5.73 & 0.03 & 8.75 & 9.73 & 0.00 \\
\hline Tyrosols & & & & & & \\
\hline ph-etOH-4'-S & 13.96 & 6.11 & 0.00 & 7.61 & 5.69 & 0.00 \\
\hline 3',4'-diOH-ph-etOH & 13.52 & 3.90 & 0.03 & 13.74 & 12.12 & 0.00 \\
\hline 3'-OH-ph-etOH-4'-S & 2.68 & - & -0.03 & 7.35 & - & 0.00 \\
\hline 4'-OH-ph-etOH-3'-S & 5.59 & - & 0.02 & 0.87 & - & 0.00 \\
\hline Benzoic acids & & & & & & \\
\hline $\mathrm{BA}$ & - & - & 0.00 & - & - & 0.00 \\
\hline 2-OH-BA & 7.68 & - & 0.05 & 19.42 & - & -0.35 \\
\hline 3-OH-BA & - & - & 0.00 & - & - & 0.00 \\
\hline 4-OH-BA & 5.25 & - & 0.00 & 1.65 & - & 0.00 \\
\hline 2,3-diOH-BA & 2.87 & - & 0.00 & 1.07 & - & 0.00 \\
\hline 2,4/2,6-diOH-BA* & 4.91 & 6.73 & 0.15 & 12.27 & 4.20 & 0.00 \\
\hline 2,5-diOH-BA & 2.57 & - & 0.03 & 0.50 & - & 0.01 \\
\hline 2,6-diOH-BA & 2.72 & 0.89 & 0.05 & 0.67 & 19.68 & 0.00 \\
\hline 3,4-diOH-BA & 7.31 & - & 0.02 & 4.10 & - & 0.00 \\
\hline 3,5-diOH-BA & 13.52 & - & 0.00 & 16.87 & - & 0.00 \\
\hline 2,3,4-triOH-BA & 10.03 & 4.78 & 0.06 & 7.78 & 2.02 & 0.03 \\
\hline
\end{tabular}




\begin{tabular}{|c|c|c|c|c|c|c|}
\hline 2-OH-4-meO-BA & 2.80 & 0.24 & 0.00 & 1.30 & 0.67 & 0.00 \\
\hline $3-\mathrm{OH}-\mathrm{BA}-4-\mathrm{S}$ & 0.92 & 1.66 & 0.04 & 19.07 & 12.27 & 0.06 \\
\hline 4-OH-BA-3-S & 3.39 & 4.17 & 0.00 & 6.82 & 0.29 & 0.05 \\
\hline 4-OH-BA-3-GlcUA & 9.32 & 11.05 & -0.05 & 1.88 & 2.41 & 0.05 \\
\hline 4-OH-3,5-dimeO-BA & 15.57 & - & 0.00 & 0.51 & - & 0.00 \\
\hline 3,4,5-triOH-Benz-OEt & 2.84 & 5.80 & 0.00 & 2.84 & 5.23 & 0.00 \\
\hline 3,4,5-triOH-BA & 1.45 & 6.83 & 0.00 & 0.68 & 14.62 & 0.03 \\
\hline $3-\mathrm{OH}-4-\mathrm{meO}-\mathrm{BA}-5-\mathrm{S}$ & 3.27 & 3.25 & 0.00 & 0.29 & 3.57 & 0.00 \\
\hline 4-OH-3-meO-BA & 19.23 & 16.62 & 0.00 & 7.79 & 16.43 & 0.00 \\
\hline 3-meO-BA-4-S & 1.55 & - & 0.10 & 1.80 & - & 0.00 \\
\hline 4-meO-BA-3-S & 6.06 & 6.94 & 0.12 & 3.06 & 1.12 & 0.00 \\
\hline \multicolumn{7}{|l|}{ Hippuric acids } \\
\hline $\mathrm{HA}$ & 0.73 & - & 0.05 & 1.69 & - & 0.00 \\
\hline 2'-OH-HA & 2.80 & 3.88 & 0.00 & 19.95 & 17.22 & 0.00 \\
\hline 3'-OH-HA & 12.63 & 14.88 & 0.00 & 4.09 & 11.47 & 0.00 \\
\hline $4^{\prime}-\mathrm{OH}-\mathrm{HA}$ & 10.21 & 6.69 & 0.02 & 12.94 & 6.43 & 0.00 \\
\hline$\alpha-\mathrm{OH}-\mathrm{HA}$ & - & - & 0.03 & - & - & 0.00 \\
\hline \multicolumn{7}{|l|}{ Cinnamic acids } \\
\hline $\mathrm{CA}$ & 3.22 & 5.97 & -0.05 & 2.77 & 13.69 & 0.00 \\
\hline 3',4'-diOH-CA & 19.93 & - & 0.07 & 15.54 & - & 0.00 \\
\hline 3'-OH-CA-4'-S & 11.41 & 0.15 & 0.05 & 0.50 & 0.93 & 0.00 \\
\hline $4^{\prime}-\mathrm{OH}-\mathrm{CA}-3^{\prime}-\mathrm{S}$ & 3.80 & 0.85 & 0.04 & 0.32 & 2.61 & 0.00 \\
\hline 3'-OH-CA-4'-GlcUA & 13.57 & - & 0.02 & 7.61 & - & 0.00 \\
\hline $4^{\prime}-\mathrm{OH}-\mathrm{CA}-3^{\prime}-\mathrm{GlcUA}$ & 0.25 & - & 0.39 & 1.89 & - & 0.00 \\
\hline 4'-OH-3'-meO-CA & 3.45 & 19.85 & 0.00 & 0.93 & 4.88 & 0.00 \\
\hline 3'-meO-CA-4'-S & 2.47 & 2.11 & 0.04 & - & - & 0.00 \\
\hline 3'-meO-CA-4'-GlcUA & 1.84 & 6.11 & 0.00 & 3.30 & 1.88 & 0.00 \\
\hline 3'-OH-4'-meO-CA & - & - & 0.00 & - & - & 0.00 \\
\hline $4^{\prime}-\mathrm{meO}-\mathrm{CA}-3^{\prime}-\mathrm{S}$ & 1.43 & 8.70 & 0.00 & 3.88 & 4.36 & 0.00 \\
\hline 4'-meO-CA-3'-GlcUA & 4.56 & 4.73 & 0.00 & 2.77 & 1.22 & 0.00 \\
\hline 4-CQA & 17.53 & 8.60 & 0.00 & 1.38 & 15.43 & 0.00 \\
\hline 4/5-CQA* & 13.61 & 18.54 & 0.00 & 4.11 & 0.24 & 0.00 \\
\hline 3-FQA & 18.94 & 18.95 & 0.00 & 2.62 & 0.65 & 0.02 \\
\hline 4-FQA & 8.37 & 10.12 & 0.00 & 10.71 & 11.35 & 0.00 \\
\hline $4^{\prime}-\mathrm{OH}-3^{\prime}, 5^{\prime}$-dimeO-CA & 3.17 & 0.87 & 0.00 & 11.30 & 0.05 & 0.00 \\
\hline $3 '-\mathrm{OH}-\mathrm{CA}$ & 16.61 & 12.64 & 0.00 & 13.13 & - & 0.00 \\
\hline 4'-OH-CA & 2.99 & - & 0.00 & 1.89 & - & 0.00 \\
\hline $2^{\prime}-\mathrm{OH}-\mathrm{CA}$ & 2.40 & 8.15 & 0.00 & 3.20 & 1.39 & 0.00 \\
\hline CA-4'-S & - & - & 0.05 & - & - & 0.00 \\
\hline CA-4'-GlcUA & 0.38 & 1.32 & 0.00 & 7.56 & 0.30 & 0.00 \\
\hline \multicolumn{7}{|l|}{ Phenylacetic acids } \\
\hline $\mathrm{PA}$ & - & - & 0.04 & - & - & 0.00 \\
\hline 3'-OH-PA & - & - & 0.00 & - & - & 0.00 \\
\hline $3^{\prime}, 4^{\prime}$-diOH-PA & - & - & 0.00 & - & - & 0.00 \\
\hline $3^{\prime}-\mathrm{meO}-\mathrm{PA}-4^{\prime}-\mathrm{S}$ & 0.56 & 0.73 & -0.01 & 9.69 & 7.07 & -0.02 \\
\hline \multicolumn{7}{|l|}{ Phenylpropionic acids } \\
\hline 2-(4'-OH-ph-O)-PrA & - & - & 0.00 & - & - & 0.00 \\
\hline $3-\left(2^{\prime}-\mathrm{OH}-\mathrm{ph}\right)-\mathrm{PrA}$ & 1.30 & - & 0.00 & 0.13 & - & 0.00 \\
\hline 3-(3'-OH-ph)-PrA & 6.06 & - & & 9.24 & - & 0.00 \\
\hline $3-\left(2^{\prime}, 3^{\prime}-\mathrm{diOH}-\mathrm{ph}\right)-\mathrm{PrA}$ & 4.05 & 4.46 & 0.02 & 1.34 & 0.48 & 0.00 \\
\hline $3-\left(2^{\prime}, 4^{\prime}-\mathrm{diOH}-\mathrm{ph}\right)-\mathrm{PrA}$ & 6.23 & 2.43 & 0.29 & 9.95 & 14.52 & 0.00 \\
\hline $3-\left(3^{\prime}, 4^{\prime}-\mathrm{diOH}-\mathrm{ph}\right)-\mathrm{PrA}$ & - & - & 0.02 & - & - & 0.00 \\
\hline $3-\left(3^{\prime}, 5^{\prime}-\mathrm{diOH}-\mathrm{ph}\right)-\mathrm{PrA}$ & 3.61 & - & 0.00 & 2.72 & - & 0.00 \\
\hline $3-\left(4^{\prime}-\mathrm{OH}-\mathrm{ph}\right)-2-\mathrm{OH}-\mathrm{PrA}$ & 4.75 & - & & 3.38 & - & 0.00 \\
\hline 3-(4'-OH-3'-meO-ph)-PrA & 12.00 & - & 0.00 & 18.37 & - & 0.00 \\
\hline 3-(4'-OH-ph)-PrA-3'-GlcUA & - & - & 0.00 & - & - & 0.00 \\
\hline $3-\left(4^{\prime}-\mathrm{OH}-\mathrm{ph}\right)-\mathrm{PrA}-3^{\prime}-\mathrm{S}$ & 3.88 & - & 0.04 & 9.02 & - & 0.00 \\
\hline $3-\left(3^{\prime}-\mathrm{meO}-\mathrm{ph}\right)-\mathrm{PrA}-4^{\prime}-\mathrm{S}$ & 12.33 & 19.98 & 0.00 & 2.16 & 10.86 & 0.00 \\
\hline 3-(3'-meO-ph)-PrA-4'-GlcUA & 15.87 & - & 0.00 & 1.19 & - & 0.00 \\
\hline 3-(4'-meO-ph)-PrA-3'-S & 16.64 & 0.00 & -0.07 & 5.85 & 5.73 & -0.06 \\
\hline 3-(4'-meO-ph)-PrA-3'-GlcUA & 3.10 & - & 0.02 & 10.94 & - & 0.03 \\
\hline \multicolumn{7}{|l|}{ Valerolactones } \\
\hline $5-\left(3^{\prime}-\mathrm{OH}-\mathrm{ph}\right)-\gamma-\mathrm{VL}-4^{\prime}-\mathrm{S}$ & 0.11 & 1.90 & -0.05 & 0.09 & 0.82 & 0.00 \\
\hline \multicolumn{7}{|l|}{ Stilbenes } \\
\hline diOH-res & 5.89 & 1.67 & 0.11 & 2.36 & 1.54 & 0.15 \\
\hline t-Res-4'-GlcUA & 0.05 & 3.15 & 0.00 & 3.25 & 5.80 & 0.00 \\
\hline t-Res-3-GlcUA & 1.87 & - & 0.03 & 0.75 & - & 0.00 \\
\hline c-Res-4'/3-GlcUA* & 0.96 & - & -0.05 & 0.50 & - & 0.00 \\
\hline c-Res-3-GlcUA & 1.26 & 0.16 & 0.00 & 3.23 & 9.29 & 0.00 \\
\hline t-Res-3-S & 0.08 & 1.16 & 0.16 & 0.77 & 1.36 & 0.00 \\
\hline
\end{tabular}

*Mix of two isomers 
Table S4. Coefficient of Variation (CV) Expressed as Percentage (\%) Between Replicates for Recovery and Matrix Effect (ME) of Compounds Studied in Plasma and Urine. For Abbreviations See Table S1.

\begin{tabular}{|c|c|c|c|c|}
\hline \multirow{2}{*}{ Compounds } & \multicolumn{2}{|c|}{ Urine } & \multicolumn{2}{|c|}{ Plasma } \\
\hline & Recovery $(\mathrm{CV}, \%)$ & $\mathrm{ME}(\mathrm{CV}, \%)$ & Recovery $(\mathrm{CV}, \%)$ & $\mathrm{ME}(\mathrm{CV}, \%)$ \\
\hline \multicolumn{5}{|l|}{ Flavanols } \\
\hline $\mathrm{EC}$ & 9 & 8 & 8 & 8 \\
\hline EC-3'-S & 11 & 10 & 14 & 9 \\
\hline 3'-ME-EC & 12 & 12 & 9 & 12 \\
\hline 4'-ME-EC & 5 & 10 & 7 & 7 \\
\hline Procyanidin A2 & 12 & 5 & 10 & 7 \\
\hline \multicolumn{5}{|l|}{ Flavanones } \\
\hline Naringenin-4'-GlcUA & 11 & 13 & 13 & 14 \\
\hline Naringenin-7-GlcUA & 6 & 6 & 8 & 14 \\
\hline Hesperetin-3'-GlcUA & 7 & 12 & 14 & 15 \\
\hline Hesperetin-7-S & 14 & 12 & 14 & 12 \\
\hline Hesperetin & 10 & 12 & 14 & 13 \\
\hline \multicolumn{5}{|l|}{ Flavones } \\
\hline Flavone & 15 & 14 & 13 & 11 \\
\hline \multicolumn{5}{|l|}{ Flavonols } \\
\hline Quercetin & 9 & 12 & 13 & 11 \\
\hline Quercetin-3-S & 7 & 8 & 13 & 8 \\
\hline Quercetin-3-GlcUA & 8 & 14 & 9 & 9 \\
\hline Quercetin-7-GlcUA & 6 & 8 & 11 & 11 \\
\hline Kaempferol-3-GlcUA & 6 & 7 & 6 & 7 \\
\hline Myricetin & 12 & 15 & 10 & 13 \\
\hline Morin & 14 & 9 & 13 & 7 \\
\hline \multicolumn{5}{|l|}{ Dihydrochalcones } \\
\hline Phloretin & 13 & 10 & 12 & 10 \\
\hline \multicolumn{5}{|l|}{ Isoflavones } \\
\hline Equol & 12 & 14 & 7 & 15 \\
\hline Equol-7-GlcUA & 6 & 8 & 6 & 6 \\
\hline Equol-4'-S & 7 & 8 & 8 & 7 \\
\hline Daidzein & 9 & 14 & 4 & 14 \\
\hline \multicolumn{5}{|l|}{ Lignans } \\
\hline SECO & 9 & 14 & 15 & 10 \\
\hline Enterodiol & 14 & 5 & 4 & 5 \\
\hline Enterolactone-GlcUA & 8 & 12 & 15 & 11 \\
\hline Enterolactone-S & 7 & 13 & 14 & 11 \\
\hline Enterolactone & 11 & 8 & 14 & 14 \\
\hline Benzene diols and triols & & & & \\
\hline 1,2-diOH-4-Me-Benz & 5 & 8 & 14 & 13 \\
\hline 2-OH-4/5-Me-Benz-1-S* & 14 & 14 & 6 & 6 \\
\hline 2-OH-Benz-1-GlcUA & 10 & 9 & 7 & 10 \\
\hline 2,6-diOH-Benz-1-S & - & - & 15 & 13 \\
\hline 2-OH-3/6-meO-Benz-1-S* & - & - & 8 & 10 \\
\hline 3-OH-2-meO-Benz-1-S & - & - & 13 & 13 \\
\hline Benzaldehydes & & & & \\
\hline 3,4-diOH-BAL & 10 & 9 & 8 & 9 \\
\hline 4-OH-BAL & 6 & 9 & 8 & 14 \\
\hline 4-OH-3-meO-BAL & 7 & 14 & 5 & 7 \\
\hline Hydroxycoumarins & & & & \\
\hline 7,8-diOH-CM & 11 & 9 & 9 & 12 \\
\hline 3,8-diOH-Uro & 7 & 7 & 12 & 11 \\
\hline 3-OH-Uro & 15 & 8 & 15 & 14 \\
\hline Tyrosols & & & & \\
\hline ph-etOH-4'-S & - & - & 14 & 9 \\
\hline 3',4'-diOH-ph-etOH & 14 & 9 & 11 & 11 \\
\hline 3'-OH-ph-etOH-4'-S & 13 & 12 & 9 & 8 \\
\hline 4'-OH-ph-etOH-3'-S & 9 & 14 & 11 & 12 \\
\hline Benzoic acids & & & & \\
\hline $\mathrm{BA}$ & 11 & 14 & 6 & 13 \\
\hline 2-OH-BA & 6 & 8 & 6 & 9 \\
\hline 3-OH-BA & 14 & 12 & 11 & 9 \\
\hline 4-OH-BA & 7 & 13 & 12 & 13 \\
\hline 2,3-diOH-BA & 7 & 15 & 7 & 9 \\
\hline 2,4/2,6-diOH-BA* & 6 & 13 & 8 & 11 \\
\hline 2,5-diOH-BA & 7 & 14 & 13 & 11 \\
\hline 2,6-diOH-BA & 9 & 12 & 6 & 8 \\
\hline 3,4-diOH-BA & 9 & 15 & 12 & 9 \\
\hline 3,5-diOH-BA & 12 & 13 & 13 & 6 \\
\hline 2,3,4-triOH-BA & 8 & 13 & 8 & 7 \\
\hline 2-OH-4-meO-BA & 6 & 8 & 8 & 10 \\
\hline 3-OH-BA-4-S & 14 & 9 & 10 & 12 \\
\hline 4-OH-BA-3-S & 13 & 13 & 6 & 12 \\
\hline 4-OH-BA-3-GlcUA & 13 & 13 & 11 & 6 \\
\hline
\end{tabular}




\begin{tabular}{|c|c|c|c|c|}
\hline 4-OH-3,5-dimeO-BA & 14 & 14 & 14 & 11 \\
\hline 3,4,5-triOH-Benz-OEt & 8 & 7 & 6 & 12 \\
\hline 3,4,5-triOH-BA & 10 & 7 & 12 & 14 \\
\hline 3-OH-4-meO-BA-5-S & 14 & 11 & 9 & 8 \\
\hline 4-OH-3-meO-BA & 12 & 11 & 11 & 12 \\
\hline 3-meO-BA-4-S & 10 & 15 & 8 & 12 \\
\hline 4-meO-BA-3-S & 13 & 13 & 7 & 7 \\
\hline \multicolumn{5}{|l|}{ Hippuric acids } \\
\hline $\mathrm{HA}$ & - & - & 5 & 15 \\
\hline $2^{\prime}-\mathrm{OH}-\mathrm{HA}$ & - & - & 5 & 12 \\
\hline 3'-OH-HA & - & - & 9 & 12 \\
\hline $4^{\prime}-\mathrm{OH}-\mathrm{HA}$ & - & - & 6 & 14 \\
\hline$\alpha-\mathrm{OH}-\mathrm{HA}$ & 11 & 14 & 9 & 8 \\
\hline \multicolumn{5}{|l|}{ Cinnamic acids } \\
\hline $\mathrm{CA}$ & 7 & 5 & 8 & 8 \\
\hline $3^{\prime}, 4^{\prime}-\mathrm{diOH}-\mathrm{CA}$ & 12 & 13 & 9 & 11 \\
\hline 3'-OH-CA-4'-S & 13 & 9 & 7 & 10 \\
\hline 4'-OH-CA-3'-S & 5 & 7 & 10 & 10 \\
\hline 3'-OH-CA-4'-GlcUA & 11 & 14 & 12 & 12 \\
\hline 4'-OH-CA-3'-GlcUA & 11 & 13 & 14 & 9 \\
\hline 4'-OH-3'-meO-CA & 10 & 14 & 10 & 10 \\
\hline 3'-meO-CA-4'-S & 7 & 14 & 9 & 13 \\
\hline 3'-meO-CA-4'-GlcUA & 8 & 11 & 5 & 12 \\
\hline 3'-OH-4'-meO-CA & 7 & 7 & 6 & 8 \\
\hline $4^{\prime}-\mathrm{meO}-\mathrm{CA}-3^{\prime}-\mathrm{S}$ & 7 & 13 & 8 & 7 \\
\hline 4'-meO-CA-3'-GlcUA & 15 & 13 & 14 & 12 \\
\hline 4-CQA & 14 & 12 & 11 & 15 \\
\hline 4/5-CQA* & 12 & 12 & 12 & 6 \\
\hline 3-FQA & 9 & 6 & 7 & 6 \\
\hline 4-FQA & 6 & 8 & 6 & 7 \\
\hline $4^{\prime}-\mathrm{OH}-3^{\prime}, 5^{\prime}$-dimeO-CA & 10 & 8 & 12 & 9 \\
\hline $3^{\prime}-\mathrm{OH}-\mathrm{CA}$ & - & - & 12 & 12 \\
\hline $4^{\prime}-\mathrm{OH}-\mathrm{CA}$ & 7 & 6 & 11 & 9 \\
\hline $2^{\prime}-\mathrm{OH}-\mathrm{CA}$ & 5 & 11 & 6 & 9 \\
\hline CA-4'-S & 6 & 11 & 9 & 9 \\
\hline CA-4'-GlcUA & 15 & 13 & 9 & 8 \\
\hline \multicolumn{5}{|l|}{ Phenylacetic acids } \\
\hline $\mathrm{PA}$ & 6 & 7 & 7 & 11 \\
\hline 3'-OH-PA & 7 & 14 & 11 & 12 \\
\hline 3',4'-diOH-PA & 11 & 10 & 6 & 8 \\
\hline $3^{\prime}-\mathrm{meO}-\mathrm{PA}-4^{\prime}-\mathrm{S}$ & 14 & 11 & 8 & 10 \\
\hline \multicolumn{5}{|l|}{ Phenylpropionic acids } \\
\hline 2-(4'-OH-ph-O)-PrA & 7 & 13 & 7 & 8 \\
\hline 3-(2'-OH-ph)-PrA & 5 & 6 & 6 & 7 \\
\hline $3-\left(3^{\prime}-\mathrm{OH}-\mathrm{ph}\right)-\mathrm{PrA}$ & 9 & 11 & 13 & 14 \\
\hline $3-\left(2^{\prime}, 3^{\prime}-\mathrm{diOH}-\mathrm{ph}\right)-\mathrm{PrA}$ & 8 & 9 & 5 & 6 \\
\hline $3-\left(2^{\prime}, 4^{\prime}-\mathrm{diOH}-\mathrm{ph}\right)-\mathrm{PrA}$ & 11 & 14 & 12 & 13 \\
\hline $3-\left(3^{\prime}, 4^{\prime}-\mathrm{diOH}-\mathrm{ph}\right)-\mathrm{PrA}$ & 8 & 9 & 7 & 9 \\
\hline $3-\left(3^{\prime}, 5^{\prime}-\mathrm{diOH}-\mathrm{ph}\right)-\mathrm{PrA}$ & 6 & 11 & 7 & 14 \\
\hline 3-(4'-OH-ph)-2-OH-PrA & 12 & 13 & 8 & 14 \\
\hline 3-(4'-OH-3'-meO-ph)-PrA & 10 & 6 & 12 & 12 \\
\hline 3-(4'-OH-ph)-PrA-3'-GlcUA & 8 & 8 & 8 & 11 \\
\hline 3-(4'-OH-ph)-PrA-3'-S & 14 & 8 & 9 & 7 \\
\hline 3-(3'-meO-ph)-PrA-4'-S & 13 & 12 & 7 & 10 \\
\hline 3-(3'-meO-ph)-PrA-4'-GlcUA & 6 & 10 & 9 & 11 \\
\hline 3-(4'-meO-ph)-PrA-3'-S & 7 & 14 & 12 & 12 \\
\hline 3-(4'-meO-ph)-PrA-3'-GlcUA & 11 & 12 & 8 & 12 \\
\hline \multicolumn{5}{|l|}{ Valerolactones } \\
\hline 5-(3'-OH-ph)- $\gamma-\mathrm{VL}-4^{\prime}-\mathrm{S}$ & 6 & 4 & 5 & 8 \\
\hline \multicolumn{5}{|l|}{ Stilbenes } \\
\hline diOH-res & 7 & 7 & 7 & 9 \\
\hline t-Res-4'-GlcUA & 11 & 14 & 11 & 11 \\
\hline t-Res-3-GlcUA & 5 & 7 & 8 & 7 \\
\hline c-Res-4'/3-GlcUA* & 6 & 8 & 6 & 8 \\
\hline c-Res-3-GlcUA & 6 & 7 & 6 & 8 \\
\hline t-Res-3-S & 8 & 10 & 14 & 9 \\
\hline
\end{tabular}

*Mix of two isomers 

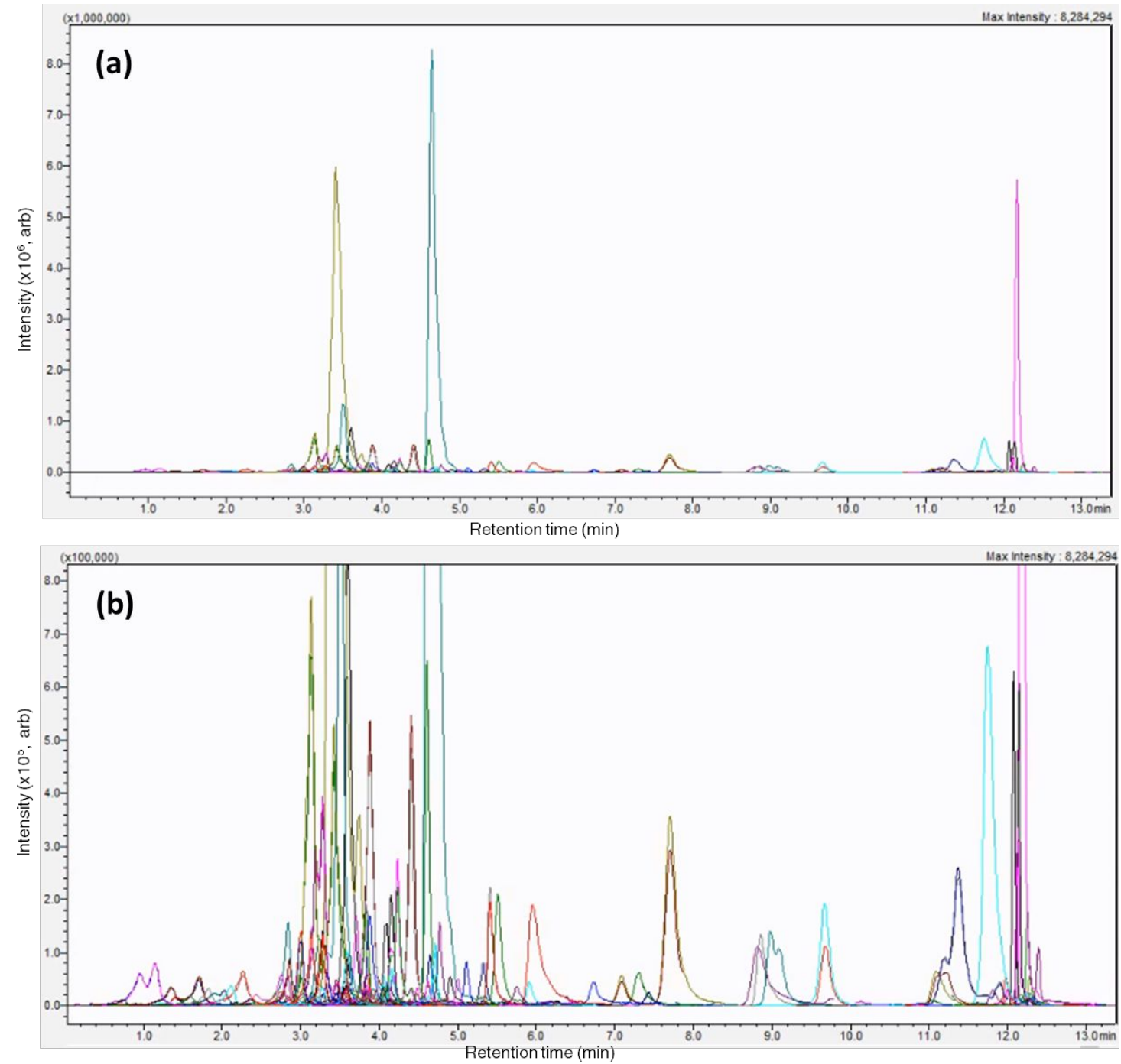

Fig S1. LC-MS/MS chromatograms of a blank urine sample spiked with the 110 standards included in the method validation, (a) showing the original intensity in the y-axis and (b) showing the same chromatogram with the intensity zoomed $\mathrm{x} 10$. 

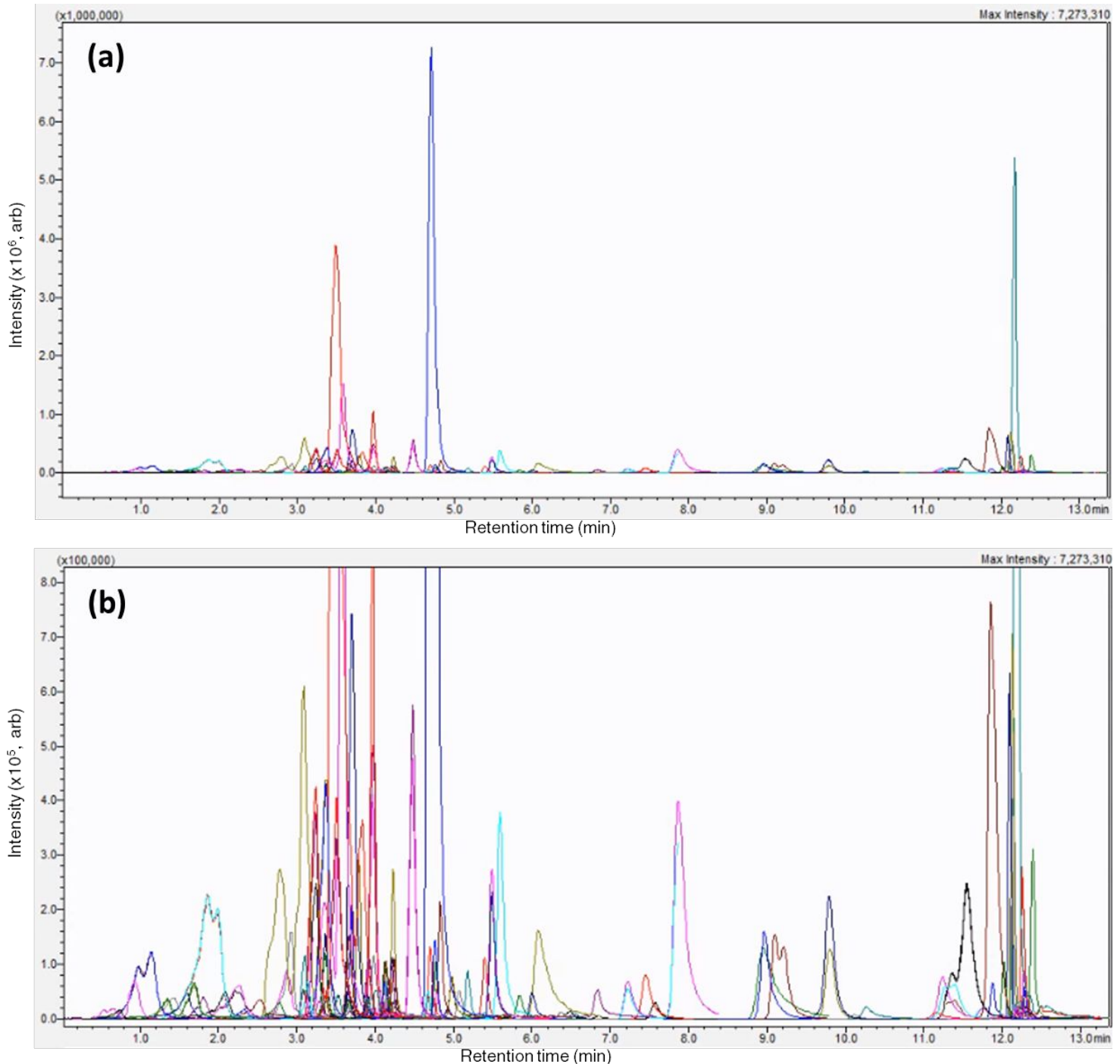

Fig S2. LC-MS/MS chromatograms of a blank plasma sample spiked with the 119 standards included in the method validation, (a) showing the original intensity in the y-axis and (b) showing the same chromatogram with the intensity zoomed $\mathrm{x} 10$. 


\section{REFERENCES}

1. Pimpao, R. C.; Ventura, M. R.; Ferreira, R. B.; Williamson, G.; Santos, C. N., Phenolic sulfates as new and highly abundant metabolites in human plasma after ingestion of a mixed berry fruit puree. Br J Nutr 2015, 113 (3), 454-63. 